\title{
A NOTE ON SUFFICIENCY IN COHERENT MODELS
}

\author{
D. BASU \\ Department of Statistics \\ Florida State University \\ Tallahassee, Florida 32306 \\ and \\ S.C. CHENG \\ Math Department, Creighton University \\ Omaha, Nebraska 68178 \\ (Paper Received February 18, 1980)
}

\begin{abstract}
Partly of an expository nature, this article brings together a number of notions related to sufficiency in an abstract measure theoretic setting. The notion of a coherent statistical mode1, as introduced by Hasegawa and Perlman [6], is studied in some details. A few results are generalized and their earlier proofs simplified. Among other things, it is shown that a coherent model can be connected in the sense of Basu [2] if and only if no splitting set (Koehn and Thomas, [7]) exists.
\end{abstract}

KEY WORDS AND PHRASES. Sufficiency, coherent model, splitting set. 1980 MATHEMATICS SUBJECT CLASSIFICATION CODES. Primary $62 \mathrm{~B} 05$.

\section{INTRODUCTION.}

This article is partly of an expository nature and is written mainly for its pedagogical interest. Given a mathematical model $(\mathcal{X}, \hat{\therefore}, P$ ) for a statistical experiment, it is of some theoretical interest to inquire whether the family of sufficient sub-s-fields (subfields) $C$ has a minimum element in some sense. It is now known that if the model is coherent in the sense of Hasegawa and Perlman [6] then such a minimum sufficient subfield exists. The case of a coherent model is 
studied in some details in this article. Among other things it is demonstrated that for a coherent model the notion of connectedness (Basu, [2]) and that of the nonexistence of a splitting set (Koehn and Thomas, [7]) coincide.

2. NOTATION AND DEFINITIONS.

The basic statistical model is denoted by $(X, A, P)$, where $X$ is the sample space, $A$ a $\sigma$-field of subsets of $X$, and $P=\left\{P_{\theta}: \quad \theta \varepsilon \theta\right\}$ a family of probability measures on $A$. By an $A$-measurable function we mean a measurable map of $(X, A)$ into $\left(R_{1}, B_{1}\right)$. Any sub- $\sigma$-field $C$ of $A$ will be referred to as a subfield. The function $\mathrm{f}$ is $\mathrm{C}$-measurable if $\mathrm{f}^{-1} B_{1}$ is contained in $C$. Given a family $\left\{A_{t}: t \varepsilon T\right\}$ of measurable sets, we write $\sigma\left\{A_{t}: t \varepsilon T\right\}$ for the subfield generated by the family of sets. Likewise, $\sigma\left\{f_{t}: t \varepsilon T\right\}$ will stand for the smallest subfield $C$ such that each $f_{t}$ is $C$-measurable. By $C V D$ we denote $\sigma(C \cup D)$, that is, the smallest subfield containing both $C$ and $D$.

A set $N$ in $A$ is $P$-null if $P_{\theta}(N)=0$ for all $\theta \varepsilon \theta$. Let $N$ denote the class of all P-null sets. For $A, B \in A$, the statement " $A=B[P]$ " means that the symmetric difference $A \Delta B$ is $P$-null. Similarly, for any two A-measurable functions $f$ and $g$, we write $f=g[P]$ to indicate that $\{x: f(x) \neq g(x)\} \in N$.

The completion $\bar{C}$ of a subfield $\mathcal{C}$ is defined as $\bar{C}=C V \sigma(N)$. Accordingly, a subfield $C$ is called complete if $N \subset C$. Let $C$ and $D$ be two subfields. We write $C \subset D[P]$ if $C \subset \bar{D}$, that is, corresponding to each set $C$ in $C$, there exists a set $D \in D$ such that $C=D[P]$. If $C \subset D[P]$ and $D \subset C[P]$, then $C=D[P]$. By a P-essentially $\bar{C}$-measurable function we mean a function $f$ such that $f^{-1}{ }_{1} \subset C[P]$, i.e., $f$ is $\bar{C}$-measurable. This is also equivalent to the statement that there exists a $C$-measurable function $g$ such that $f=g[P]$. An A-measurable function is P-integrable if $\int_{\lambda}|\mathrm{f}| \mathrm{d} \mathrm{P}_{\theta}<\infty$ for all $\theta \varepsilon \theta$.

DEFINITION 1. (Halmos and Savage, [5]). The statistical model (X, A, P) is called dominated if every probability measure in $P$ is absolutely continuous with respect to a fixed $\sigma$-finite measure $\lambda$ on $A$.

In this case, we say that the family is dominated by $\lambda$ and write $P \ll \lambda$. 
DEFINITION 2. (Basu and Ghosh, [3]). The statistical model $(X, A, P)$ is called discrete if

(i) each $\mathrm{P}_{\theta}$ is a discrete probability measure,

(ii) $A$ is the class of all subsets of $X$, and

(iii) for each $x \in X$, there exists a $\theta \varepsilon \theta$ such that $P_{\theta}(\{x\})>0$.

Condition (iii) implies that the empty set is the only P-null set. A discrete model with a countable sample space $X$ is clearly dominated. If $P$ is countable, then the model is dominated. We assume that both $X$ and $P$ are uncountable. In this case, the model will be undominated. As we shall see in the next section, dominated and discrete models are particular cases of what Hasegawa and Perlman [6] called a coherent model.

Finally, let us state the notion of sufficiency as follows. A subfield $C$ is sufficient with respect to the model $(X, A, P)$ if, corresponding to each $A$ in $A$, there exists a $C$-measurable function $I_{A}^{\dagger}$ such that $I_{A}^{\dagger}=E_{\theta}\left(I_{A} \mid C\right)\left[P_{\theta}\right]$ for all $\theta \varepsilon \theta$. A subfield $C$ is pairwise sufficient with respect to $(X, A, P)$ if, for each $A$ in $A$ and each pair $\theta_{1}, \theta_{2} \varepsilon \theta$, there exists a $C$-measurable function $I_{A}^{*}$ such that $I_{\dot{A}}^{*}=E_{\theta_{i}}\left(I_{A}^{*} \mid C\right)\left[P_{\theta_{i}}\right]$ for $i=1$, 2. (The function $I_{A}^{*}$ may depend on $\theta_{1}$ and $\theta_{2}$. ) 3. COHERENT STATISTICAL MODEL.

Let $F$ denote the class of all measurable functions $f: X \rightarrow[0,1]$ and let

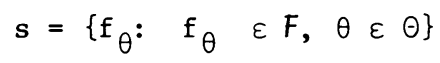

be a collection of members of $F$ that is indexed by $\theta$. Let $S=\{s\}$ be the family of all such collections s.

DEFINITION 3. A member $\left\{\mathrm{f}_{\theta}\right\}$ of $S$ is said to be pairwise coherent if, for every pair $\theta_{1}, \theta_{2}$ in $\theta$, there exists a function $f_{12}$ in $F$ such that $f_{\theta_{1}}=f_{12}$ $\left.{ }^{\left[P_{\theta_{i}}\right.}\right]$ for $\mathbf{i}=1,2$.

DEFINITION 4. A member $\left\{\mathrm{f}_{\theta}\right\}$ of $S$ is said to be countably coherent if, for every countable subfamily $\theta_{0}=\left\{\theta_{1}, \theta_{2}, \ldots\right\}$ of $\theta$, there exists a function $f_{0}$ in $F$ such that $f_{\theta_{i}}=f_{0}\left[P_{\theta_{i}}\right]$ for all $i=1,2, \ldots$.

DEFINITION 5. A member $\left\{\mathrm{f}_{\theta}\right\}$ of $S$ is said to be coherent if there exists a function $f$ in $F$ such that $f_{\theta}=f\left[P_{\theta}\right]$ for all $\theta \varepsilon \theta$. 
DEFINITION 6. (Hasegawa and Perlman, [6]). The statistical model (X, A, P) is said to be coherent if every countably coherent member of $S$ is coherent.

In the following lemma, we show that the notions of pairwise coherence and countable coherence do coincide.

LEMMA 1. If $\left\{f_{\theta}\right\}$ is a pairwise coherent member of $S$, then it is countably coherent.

PROFF. Choose and fix a countable subfamily $\theta_{0}=\left\{\theta_{1}, \theta_{2}, \ldots\right\}$ of $\theta_{\text {. For }}$ each pair $\theta_{i}, \theta_{j}$ in $\theta_{0}$, there exists a function $f_{i j}$ in $F$ such that

$$
f_{i j}=f_{\theta_{i}}\left[P_{\theta_{i}}\right] \text { and } f_{i, i}=f_{\theta_{j}}\left[P_{\theta}\right] \text {. }
$$

Let

$$
g_{i}=\sup _{j} f_{i j}, h_{j}=\inf _{i} f_{i j} \text {, and } f=\underset{i}{\inf } \sup _{j} f_{i j} \text {. }
$$

For each fixed $i$, the functions $f_{i 1}, f_{i 2}, \ldots$ are $P_{\theta_{i}}$-equivalent to $f_{\theta_{i}}$. The supremum of a countable number of $\mathrm{P}_{\theta_{i}}$-equivalent functions is also $\mathrm{P}_{\theta_{i}}$-equivalent to those functions. Thus, $g_{i}=f_{\theta_{i}}\left[P_{\theta_{i}}\right]$. Likewise, for each fixed $j$, we have $h_{j}=f_{\theta_{j}}\left[P_{\theta_{j}}\right]$. Therefore, it follows that $g_{n}=h_{n}=f_{\theta_{n}}\left[P_{\theta_{n}}\right]$ for $n=1,2, \ldots$ observe that $h_{\mathrm{n}} \leq \mathrm{f} \leq \mathrm{g}_{\mathrm{n}}$ for all $\mathrm{n}$. It then follows that

$$
f=g_{n}=h_{n}=f_{\theta_{n}}\left[P_{\theta_{n}}\right] \text { for } n=1,2, \ldots .
$$

Hence, $\left\{f_{\theta}\right\}$ is countably coherent.

In general, the notions of pairwise coherence and coherence do not coincide as the following example shows.

EXAMPLE 1. (Pitcher, [10]). Let $X$ be the unit interval $[0,1], A$ the $\sigma$-field of Borel subsets of $X$, and $P$ the family of all probability measures on A which are either degenerate at a single point of $X$ or else are absolutely continuous with respect to the Lebesgue measure. For each $P_{\theta}$ in $P$ and each $x \in X$, define $f_{\theta}(x)=P_{\theta}(\{x\})$. Then $\left\{f_{\theta}\right\}$ is pairwise coherent, but not coherent.

In this model, no proper subfield of $A$ can be sufficient. To see this, let $C$ be an arbitrary sufficient subfield and let $P_{x}$ be the probability measure degenerate at $x$. Then it follows from the sufficiency of $C$ that, for all $A$ in 
A, there exists a C-measurable function $I_{A}^{\dagger}$ such that

$$
I_{A}^{\dagger}(x)=\int_{X} I_{A}^{\dagger} d P_{x}=\int_{X} I_{A} d P_{x}=I_{A}(x) \text {. }
$$

Let $C=\left\{x: \quad I_{A}^{+}(x)=1\right\}$. Then $A=C \varepsilon C$. Hence, $C=A$.

We now show that the model $(X, A, P)$ is coherent if it is either dominated or discrete.

LEMMA 2. (Hasegawa and Perlman, [6]). If $(X, A, P)$ is dominated, then it is coherent.

PROOF. Since $P$ is dominated, it follows that there is a countable subfamily $\theta_{0}=\left\{\theta_{1}, \theta_{2}, \ldots\right\}$ of $\theta$ such that $P_{0}=\left\{P_{\theta}: \theta \varepsilon \theta_{0}\right\}$ is equivalent to $P$. Suppose that $\left\{\mathrm{f}_{\theta}\right\}$ is countably coherent. Then there exists a function $\mathrm{f}_{0} \varepsilon F$ such that $\mathrm{f}_{0}=\mathrm{f}_{\theta_{\mathrm{n}}}\left[\mathrm{P}_{\theta_{\mathrm{n}}}\right]$ for $\mathrm{n}=1,2, \ldots$. We now show that $\mathrm{f}_{0}=\mathrm{f}_{\theta}\left[\mathrm{P}_{\theta}\right]$ for a11 $\theta \varepsilon 0$, so $\left\{f_{\theta}\right\}$ is coherent. For each $\theta \varepsilon \theta$, consider $\theta_{1}=\theta_{0} U\{\theta\}$. Then there exists a function $\mathrm{f}_{1} \varepsilon F$ such that $\mathrm{f}_{1}=\mathrm{f}_{\theta_{\mathrm{n}}}\left[\mathrm{P}_{\theta_{\mathrm{n}}}\right]$ for $\mathrm{n}=1,2, \ldots$. Thus, $\mathrm{f}_{0}=\mathrm{f}_{1}\left[\mathrm{P}_{0}\right]$ and so $\mathrm{f}_{0}=\mathrm{f}_{1}[\mathrm{P}]$. Hence, $\mathrm{f}_{0}=\mathrm{f}_{1}=\mathrm{f}_{\theta}\left[\mathrm{P}_{\theta}\right]$ as required.

LEMMA 3. If $(X, A, P)$ is discrete, then it is coherent.

PROOF. Let $\left\{f_{\theta}\right\}$ be pairwise coherent. We must show that it is also coherent. For each $\theta \varepsilon \theta$, let $\mathrm{S}_{\theta}=\left\{\mathrm{x} \varepsilon \mathrm{X}: \mathrm{P}_{\theta}(\{\mathrm{x}\})>0\right\}$ denote the countable support of $\mathrm{P}_{\theta}$. For each pair $\theta_{1}, \theta_{2}$ in $\theta$, there exists a function $\mathrm{f}_{12}$ in $F$ such that $\mathrm{f}_{12}=$ $\mathrm{f}_{\theta_{i}}\left[\mathrm{P}_{\theta_{i}}\right]$ for $i=1,2$. Thus, we have

$$
\mathrm{f}_{\theta_{i}}(\mathrm{x})=\mathrm{f}_{12}(\mathrm{x}) \text { for all } \mathrm{x} \in \mathrm{s}_{\theta_{i}}, \mathbf{i}=1,2 \text {. }
$$

Hence, $\mathrm{f}_{\theta_{1}}(\mathrm{x})=\mathrm{f}_{\theta_{2}}(\mathrm{x})$ for all $\mathrm{x} \varepsilon \mathrm{s}_{\theta_{1}} \bigcap \mathrm{s}_{\theta_{2}}$. Now, choose and $\mathrm{fix} \mathrm{x} \varepsilon$. Let $\Theta_{x}=\left\{\begin{array}{lllll}\theta & \varepsilon \theta & x & \varepsilon & s_{\theta}\end{array}\right\}$. Let $\theta_{0}$ be a member of $\theta_{x}$. Note that $x \varepsilon s_{\theta_{0}} \bigcap$ s for all $\theta \varepsilon \Theta_{x^{*}}$. In view of (1), we have $f_{\theta_{0}}(x)=f_{\theta}(x)$ for all $\theta \varepsilon \theta_{x}$, that is, $f_{\theta}(x)$ is constant in $\theta \varepsilon \theta_{x}$ (for this prefixed $x$ ). Let $c_{x}$ be the common value of $\mathrm{f}_{\theta}$ for $\theta \varepsilon \Theta_{\mathrm{x}}$, evaluated at $\mathrm{x}$, that is, $\mathrm{f}_{\theta}(\mathrm{x})=\mathrm{c}_{\mathrm{x}}$ for $\mathrm{a} 11 \mathrm{x} \varepsilon \mathrm{X}$. Since $\mathrm{x}$ is arbitrary, define a function $f$ by $f(x)=c_{x}$ for all $x \varepsilon x$. clearly, $f=f_{\theta}$ $\left[\mathrm{P}_{\theta}\right]$ for all $\theta \in \Theta$ as required.

That the coherent case is not exhausted by the dominated and discrete cases is shown in the following example. 
EXAMPLE 2. Let $\left(X_{1}, A_{1}, P_{1}\right)$ be a non-discrete dominated model, let $\left(X_{2}, A_{2}\right.$, $P_{2}$ ) be an undominated discrete model, where $X_{1}$ and $X_{2}$ are disjoint, $P_{1}=$ $\left\{P_{\theta}: \theta \varepsilon \Theta_{1}\right\}$, and $P_{2}=\left\{P_{\theta}: \theta \varepsilon \theta_{2}\right\}$. Let $X=X_{1} \cup X_{2}, A=\left\{A \subset X: A X_{1} \varepsilon A_{i}\right.$ for $i=1,2\}$, and extend $P_{1}$ and $P_{2}$ to $A$ by defining

$$
P_{\theta}(A)=P_{\theta}\left(A X_{i}\right) \text { for all } \theta \varepsilon \theta_{i}, i=1,2 \text {. }
$$

It follows from Lemmas 2 and 3 that both $\left(X_{1}, A_{1}, P_{1}\right)$ and $\left(X_{2}, A_{2}, P_{2}\right)$ are coherent. Now we claim that $(X, A, P)$ is also coherent, where $P=P_{1} U P_{2}=\left\{P_{\theta}: \theta \varepsilon \theta_{1} U \theta_{2}\right.$ $=\theta\}$. Suppose that $\left\{f_{\theta}: \theta \in \theta\right\}$ is pairwise coherent with respect to $(X, A, P)$. Each $f_{\theta}$, being an A-measurable function, can be written as

$$
f_{\theta}= \begin{cases}f_{\theta}^{1} & \text { on } x_{1} \\ f_{\theta}^{2} & \text { on } x_{2},\end{cases}
$$

where $f_{\theta}^{i}$ is $A_{i}$-measurable for $i=1$, 2. Since $\left\{f_{\theta}^{i}: \theta \varepsilon \theta_{i}\right\}$, being pairwise coherent with respect to $\left(X_{i}, A_{i}, P_{i}\right)$, is coherent, it follows that there exists an $A_{i}-$ measurable function $0 \leq f_{i} \leq 1$ such that

Define

$$
f_{i}=f_{\theta}^{i}\left[P_{\theta}\right] \text { for all } \theta \varepsilon \theta_{i} \text {. }
$$

$$
f= \begin{cases}f_{1} & \text { on } x_{1} \\ f_{2} & \text { on } x_{2}\end{cases}
$$

Clearly, $f$ is A-measurable. Observe that $f_{\theta}=f_{\theta}^{i}\left[P_{\theta}\right]$ for all $\theta \varepsilon \theta_{i}, i=1,2$. Therefore, $f=f_{\theta}\left[P_{\theta}\right]$ for all $\theta \varepsilon \theta$. However, it should be noted that $(X, A, P)$ is neither dominated nor discrete.

\section{SUFFICIENCY IN THE COHERENT CASE.}

For each $\theta \varepsilon \theta$, let $N_{\theta}$ denote the class of all $\mathrm{P}_{\theta}$-null sets, that is, $N_{\theta}=\left\{N \in A: P_{\theta}(N)=0\right\}$. For each subfield $C$ of $A$, define

$$
\tilde{\mathrm{c}}=\prod_{\theta \varepsilon \Theta}\left[C \vee \sigma\left(N_{\theta}\right)\right]
$$

Then $\tilde{C}$ is also a subfield of $A$. It is easy to see that a function $f$ is $\tilde{C}$-measurable 
if and only if, for each $\theta \varepsilon \theta$, there exists a $\mathcal{C}$-measurable function $\mathrm{f}_{\theta}$ such that $\mathbf{f}=\mathrm{f}_{\theta}\left[\mathrm{P}_{\theta}\right] . \quad \mathrm{clear} 1 \mathrm{y}, \mathrm{C} \subset \overline{\mathrm{C}} \subset \tilde{\mathrm{C}}$.

Let $(X, A, P)$ be a coherent model. Then, so is $\left(X, A, P_{0}\right)$ for any subfamily $P_{0}$ of $P$. However, it is not true that $(X, C, P)$ is coherent for every subfield C of A. Such an example is explicitly included in Pitcher's [9] example.

EXAMPLE 3. Let $X$ be the real line and let $B$ be the $\sigma$-field of all Borel subsets of $X$. Choose and fix a non-empty, non-Borel set $E$ that excludes the origin but is symmetric about the origin, i.e., $E=-E=\{x:-x \in E\}$. Let $\Theta$ be also the real line, and define a family $P=\left\{\mathrm{P}_{\theta}: \theta \varepsilon \Theta\right\}$ of probability measures as follows: If $\theta \varepsilon \mathrm{E}$, then $\mathrm{P}_{\theta}$ is the discrete measure allotting probabilities $1 / 2$ and $1 / 2$ to the two points $-\theta$ and $\theta$. If $\theta \notin E$, then $P_{\theta}$ is degenerate at $\theta$. We claim that $(X, B, P)$ cannot be coherent. To see this, for each $\theta \varepsilon \theta$ and each $x \varepsilon X$, define

$$
f_{\theta}(x)=P_{\theta}(\{x\})
$$

Then $\left\{\mathrm{f}_{\theta}: \theta \varepsilon \theta\right\}$ is pairwise coherent with respect to $(X, B, P)$. Suppose, on the contrary, that $\left\{\mathrm{f}_{\theta}: \theta \varepsilon \Theta\right\}$ is coherent. Then there exists a $B$-measurable function $0 \leq \mathrm{f} \leq 1$ such that $\mathrm{f}=\mathrm{f}_{\theta}\left[\mathrm{P}_{\theta}\right]$ for all $\theta \varepsilon \theta$. This implies that

$$
f(x)=\left\{\begin{array}{cc}
1 / 2 & \text { if } x \in E \\
1 & \text { if } x \notin E,
\end{array}\right.
$$

which obviously contradicts the initial supposition that $E \notin B$. However, if we consider the class $A$ of all subsets of $X$, then $(X, A, P)$ is a discrete model and hence is coherent.

In the following lemma, however, we show that if $(X, A, P)$ is a coherent model, then so is $(X, \tilde{C}, P)$ for any subfield $C$ of $A$.

LEMMA 4. If $(X, A, P)$ is coherent and $C$ is a subfield of $A$, then $(X, \tilde{C}, P)$ is coherent.

PROOF. Let $\left\{\mathrm{f}_{\theta}: \theta \varepsilon \Theta\right\}$ be pairwise coherent with respect to $(X, \tilde{C}, P)$. Then it is also pairwise coherent with respect to $(X, A, P)$. Since $(X, A, P)$ is coherent, there exists an A-measurable function $0 \leq f \leq 1$ such that $f=f_{\theta}\left[P_{\theta}\right]$ for all $\theta \varepsilon \theta$. Since each $f_{\theta}$ is $\tilde{C}$-measurable, it is seen that $f$ must be 
measurable with respect to $\tilde{\tilde{C}}=\tilde{C}$. This proves that $(X, \tilde{C}, P)$ is coherent.

Let $\tilde{P}$ denote the convex hull of $P$, that is, $\tilde{P}=\left\{Q: Q=\sum a_{i} P_{\theta_{i}}, a_{i} \geq 0\right.$, $\left.\Sigma_{a_{i}}=1, \theta_{i} \varepsilon \theta\right\}$.

LEMMA 5. $(X, A, P)$ is coherent if and only if $(X, A, \tilde{P})$ is coherent.

PROOF. The "only if" part is what needs to be proved. Let $\left\{f_{Q}: Q \in \tilde{P}\right\}$ be pairwise coherent with repsect to $(X, A, \tilde{P})$. Then the subset $\left\{f_{\theta}: \theta \varepsilon \theta\right\}$ of $\left\{f_{Q}: \dot{Q} \varepsilon \tilde{P}\right\}$ is pairwise coherent with respect to $(X, A, P)$. Since $(X, A, P)$ is coherent, there exists an A-measurable function $0 \leq f \leq 1$ such that $f=f_{\theta}\left[P_{\theta}\right]$ for all $\theta \varepsilon \theta$. We now claim that $f=f_{Q}[Q]$ for all $Q \varepsilon \tilde{P}$. Choose and $f i x Q \varepsilon \ddot{P}$. Then $Q=\sum a_{i} P_{\theta_{i}}$, where $\theta_{i} \varepsilon \theta, a_{i} \geq 0$, and $\sum a_{i}=1$. For each pair $Q, P_{\theta_{i}}$, there exists an A-measurable function $0 \leq f_{i} \leq 1$ such that

$$
f_{i}=f_{Q}[Q]
$$

and

$$
\mathrm{f}_{i}=\mathrm{f}_{\theta_{i}}=\mathrm{f}\left[\mathrm{P}_{\theta_{i}}\right]
$$

Since $\left\{P_{\theta_{i}}: i=1,2, \ldots\right\} \equiv Q$, it follows that

$$
\mathrm{f}=\mathrm{f}_{i}=\mathrm{f}_{Q}\left[\mathrm{P}_{\theta_{i}}\right] \text { for } i=1,2, \ldots .
$$

Hence, $f=f_{Q}[Q]$ as required.

LEMMA 6. Let $C$ be a subfield of $A$ such that $(X, C, P)$ is coherent. If $P$ is closed for countable convex combinations (i.e., $P=\tilde{P}$ ), then $\bar{C}=\tilde{C}$.

PROOF. Since $\bar{C} \subset \tilde{C}$, it suffices to show that $\tilde{C} \subset \bar{C}$. Let $f$ be a $\tilde{C}$-measurable function such that $0 \leq \mathrm{f} \leq 1$. Then, for each $\theta \in \theta$, there exists a $\mathcal{C}$-measurable function $0 \leq f_{\theta} \leq 1$ such that $f=f_{\theta}\left[P_{\theta}\right] . \quad$ For each pair $\theta_{1}, \theta_{2} \varepsilon \theta$, let $Q=\left(P_{\theta_{1}}+P_{\theta_{2}}\right) / 2$. Then $Q \varepsilon \tilde{P}=P$ and so $f=f_{Q}[Q]$. Since $\left\{P_{\theta_{1}}, P_{\theta_{2}}\right\} \equiv Q$, we have $f_{Q}=f=f_{\theta_{i}}\left[P_{\theta_{i}}\right]$ for $i=1,2$. Thus, we have shown that $\left\{f_{\theta}: \theta \varepsilon \theta\right\}$ is pairwise coherent with respect to $(X, C, P)$. Therefore, there exists a $C$-measurable function $0 \leq \mathrm{f}_{0} \leq 1$ such that $\mathrm{f}_{0}=\mathrm{f}_{\theta}\left[\mathrm{P}_{\theta}\right]$ for all $\theta \varepsilon \theta$, and hence $\mathrm{f}=\mathrm{f}_{0}[\mathrm{P}]$. This shows that $\tilde{C} \subset \bar{C}$.

LEMMA 7. $(X, C, P)$ is coherent if and only if $(X, \bar{C}, P)$ is coherent. 
PROOF. Let us first prove the "only if" part. Let $\left\{f_{\theta}: \theta \varepsilon \theta\right\}$ be pairwise coherent with respect to $(X, \bar{C}, P)$. For each $\theta \varepsilon \theta$, there exists a $C$-measurable function $0 \leq \mathrm{g}_{\theta} \leq 1$ such that $\mathrm{f}_{\theta}=\mathrm{g}_{\theta}\left[\mathrm{P}_{\theta}\right]$. For each pair $\theta_{1}, \theta_{2} \varepsilon \theta$, there exists a $\bar{C}$-measurable function $0 \leq \mathrm{f}_{12} \leq 1$ such that $\mathrm{f}_{12}=\mathrm{f}_{\theta_{i}}\left[\mathrm{P}_{\theta_{i}}\right]$ for $i=1$, 2. Since there is a $C$-measurable function $0 \leq g_{12} \leq 1$ such that $f_{12}=g_{12}[P]$, it follows that $g_{12}=g_{\theta_{i}}\left[P_{\theta_{i}}\right]$ for $i=1,2$. Hence, $\left\{g_{\theta}: \theta \varepsilon \theta\right\}$ is pairwise coherent with respect to $(X, C, P)$. Since $(X, C, P)$ is coherent, there exists a $C$-measurable function $0 \leq \mathrm{f} \leq 1$ such that $\mathbf{f}=\mathrm{g}_{\theta}=\mathrm{f}_{\theta}\left[\mathrm{P}_{\theta}\right]$ for all $\theta \varepsilon \theta$. Therefore, $(X, \bar{C}, P)$ is coherent.

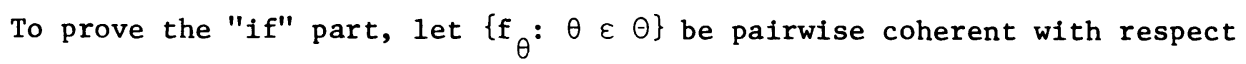
to $(X, C, P)$. Then, it is also pairwise coherent with respect to $(X, \bar{C}, P)$. Thus, there exists a $\bar{C}$-measurable function $0 \leq \overline{\mathrm{f}} \leq 1$ such that $\overline{\mathrm{f}}=\mathrm{f}_{\theta}\left[\mathrm{P}_{\theta}\right]$ for all $\theta \varepsilon \Theta$. Since there is a $C$-measurable function $0 \leq \mathrm{f} \leq 1$ such that $\mathrm{f}=\overline{\mathrm{f}}[\mathrm{P}]$, we have $\mathbf{f}=\mathbf{f}_{\theta}\left[\mathrm{P}_{\theta}\right]$ for all $\theta \varepsilon \Theta$ as required.

Under the assumption of coherence, we now prove a number of results on sufficiency.

PROPOSITION 1. Suppose that $(X, C, P)$ is coherent. If $C$ is pairwise sufficient, then $C$ is sufficient.

PROOF. Chose and fix $A \in A$. For each $\theta \varepsilon \theta$, let $0 \leq f_{\theta} \leq 1$ be a version of $\mathrm{E}_{\theta}\left(\mathrm{I}_{A} \mid C\right)$. Since $\mathcal{C}$ is pairwise sufficient, for each pair $\theta_{1}, \theta_{2} \varepsilon \theta_{\text {, there }}$ exists a $C$-measurable function $0 \leq \mathrm{f}_{12} \leq 1$ such that $\mathrm{f}_{12}=\mathrm{f}_{\theta_{i}}\left[\mathrm{P}_{\theta_{i}}\right]$ for $1=1$, 2 . Thus, $\left\{f_{\theta}: \theta \varepsilon \theta\right\}$ is pairwise coherent with respect to $(X, C, P)$. Since $(X, C, P)$ is coherent, there exists a $C$-measurable function $0 \leq \mathrm{f} \leq 1$ such that $\mathrm{f}=\mathrm{f}_{\theta}\left[\mathrm{P}_{\theta}\right]$ for all $\theta \varepsilon \Theta$. That is, $C$ is sufficient.

COROLLARY 1. Suppose that $(X, A, P)$ is coherent and $\bar{C}=\tilde{C}$. If $C$ is pairwise sufficient, then $C$ is sufficient.

PROOF. Since $(X, A, P)$ is coherent, it follows from Lemma 4 that $(X, \tilde{C}, P)$ is coherent. Since $\bar{C}=\tilde{C},(X, \bar{C}, P)$ is coherent. In view of Lemma $7,(X, C, P)$ is coherent. Consequently, the result follows from Proposition 1. 
CORALLARY 2. Let $C \subset D\left[P_{\theta_{1}}, P_{\theta_{2}}\right]$ for all pairs $\theta_{1}, \theta_{2} \varepsilon \theta$. If $C$ is pairwise sufficient and $(X, D, P)$ is coherent, then $D$ is sufficient.

PROOF. Since $C$ is pairwise sufficient and $C \subset D\left[P_{\theta_{1}}, P_{\theta_{2}}\right]$ for all $\theta_{1}, \theta_{2}$ $\varepsilon \theta$, it is easy to verify that $D$ is also pairwise sufficient. Since $(X, D, P)$ is coherent, it follows from Proposition 1 that $D$ is sufficient.

COROLLARY 3. Suppose that $(X, A, P)$ is coherent and $C$ is sufficient. If $C \subset D\left[P_{\theta_{1}}, P_{\theta_{2}}\right]$ for all $\theta_{1}, \theta_{2} \varepsilon \theta$ and $\bar{D}=\tilde{D}$, then $D$ is sufficient.

PROOF. Since $(X, A, P)$ is coherent and $\bar{D}=\tilde{D}$, it follows from Lemmas 4 and 7 that $(X, D, P)$ is coherent. In view of Corollary 2, we therefore conclude that $D$ is sufficient.

We now show that if $(X, A, P)$ is coherent, then so is $(X, C, P)$ for any sufficient subfield $C$. To this end, we shall need the following lemma.

LEMMA 8. (Pitcher, 1965). If $C$ is sufficient, then $\bar{C}=\bar{C}$.

PROOF. Let $A \in \mathcal{C}$. Since $C$ is sufficient, there exists a $C$-measurable function $I_{A}^{+}$such that $I_{A}^{+}=E_{\theta}\left(I_{A} \mid C\right)\left[P_{\theta}\right]$ for all $\theta \varepsilon \theta$. Note that $I_{A}-I_{A}^{+}$is $\tilde{C}_{-}$ measurable. Thus, for each $\theta \varepsilon \theta$, there exists a $C$-measurable function $f_{\theta}$ such that $I_{A}-I_{A}^{\dagger}=f_{\theta}\left[P_{\theta}\right]$ and so

$$
\begin{aligned}
& \int_{X}\left(I_{A}-I_{A}^{\dagger}\right){ }^{2} d P_{\theta}=\int_{X}\left(I_{A}-I_{A}^{+}\right) f_{\theta} d P_{\theta} \\
& =\int_{X} E_{\theta}\left[\left(I_{A}-I_{A}^{\dagger}\right) f_{\theta} \mid C\right] d P_{\theta} \\
& =\int_{X} f_{\theta} E_{\theta}\left(I_{A}-I_{A}^{\dagger} \mid C\right) d P \\
& =0 .
\end{aligned}
$$

Hence, $I_{A}=I_{A}^{\dagger}\left[P_{\theta}\right]$ for all $\theta \varepsilon \theta$. Since $I_{A}^{\dagger}$ is C-measurable, it follows that $A \varepsilon \bar{C}$. Therefore, $\bar{C}=\tilde{C}$.

PROPOSITION 2. If $(X, A, P)$ is coherent and $C$ is sufficient, then $(X, C, P)$ is coherent.

PROOF. Since $C$ is sufficient, $\bar{C}=\tilde{C}$ in view of Lemma 8 . Since $(X, A, P)$ is coherent, it follows from Lemma 4 and 7 that $(X, C, P)$ is coherent.

5. BASU'S THEOREM.

As before, $(X, A, P)$ is our basic model, where $P=\left\{P_{\theta}: \theta \varepsilon \theta\right\}$. Two 
probability measures $P_{\theta_{1}}$ and $P_{\theta_{2}}$ in $P$ are said to be overlapping if, for any set $A$ in $A, P_{\theta_{1}}(A)=1$ implies that $P_{\theta_{2}}(A)>0$. We write $\theta_{1} \Leftrightarrow \theta_{2}$ if $P_{\theta_{1}}$ and $P_{\theta_{2}}$ overlap. If there exists a finite number of parameter points $\theta_{1}, \theta_{2}, \ldots, \theta_{k}$ such that

$$
\theta_{1} \Leftrightarrow \theta_{2} \Leftrightarrow \quad \ldots \Leftrightarrow \theta_{\mathrm{k}} \Leftrightarrow \theta^{\prime},
$$

then we say $P_{\theta}$ and $P_{\theta}$ ' are connected. The family $P$ is called connected if every pair of probability measures in the family are connected.

THEOREM 1. (Basu, [2]). If $\mathrm{T}$ is a sufficient statistic, $P$ is connected, and $\mathrm{V}$ is a statistic which is independent of $\mathrm{T}$ for all $\theta \varepsilon \theta$, then the distribution of $\mathrm{V}$ does not depend on $\theta$.

A set $A$ in $A$ is called splitting set if there is a partition $\theta_{0}, \theta_{1}$, of $\theta$ such that

$$
P_{\theta}(A)=\left\{\begin{array}{l}
0 \text { if } \theta \varepsilon \theta_{0} \\
1 \text { if } \theta \varepsilon \theta_{1}
\end{array}\right.
$$

The above theorem has been generalized by Koehn and Thomas [7] as follows:

THEOREM 2. Let $\mathrm{T}$ be a sufficient statistic. There exists a statistic $\mathrm{V}$, independent of $\mathrm{T}$ for all $\theta \varepsilon \theta$, whose distribtuion depends on $\theta$ if and only if there exists a splitting set.

We now demonstrate that the two notions of connectedness of $P$ and the nonexistence of a splitting set are equivalent in the coherent case.

LEMMA 9. Let $(X, A, P)$ be coherent. Then $P$ is connected if and only if there exists no splitting set.

PROOF. Clearly, the connectedness of $P$ implies the nonexistence of a splitting set. For each $\theta \varepsilon \theta$, let $A_{\theta}$ be a set such that $P_{\theta}\left(A_{\theta}\right)=1$ and $P_{\theta}(B)>0$ if $\phi \neq B \subset A_{\theta}$. Suppose that $P$ is not connected. Choose and fix $\theta_{0} \varepsilon \theta$. Let $\theta_{0}=\left\{\theta \varepsilon \theta: P P_{\theta}\right.$ and $P_{\theta}$ are connected $\}$. Since $P$ is not connected, $\theta_{1}=\theta \backslash \theta_{0}$ is not empty. For each $\theta \varepsilon \theta$, let $\mathrm{f}_{\theta}=\mathrm{I}_{\mathrm{A}_{\theta}}$. It is easy to show that $\left\{\mathrm{f}_{\theta}: \theta \varepsilon \theta\right\}$ is pairwise coherent. Since $(X, A, P)$ is coherent, there exists an A-measurable 
function $0 \leq \mathrm{f} \leq 1$ such that $\mathrm{I}_{\mathrm{A}_{\theta}}=\mathrm{f}\left[\mathrm{P}_{\theta}\right]$ for all $\theta \varepsilon \theta$. Let $\mathrm{A}=\bigcup_{\theta \varepsilon \Theta_{0}} \mathrm{~A}_{\theta}$. Since $A_{\theta}$ is the support of $P_{\theta}$, it is easily seen that

$$
P_{\theta}(A)=P_{\theta}\left(A_{\theta}\right)=1 \text { for all } \theta \varepsilon \theta_{0} \text {. }
$$

Note that $A \cap A_{\theta}=\phi$ if $\theta \varepsilon \theta_{1}$. Thus,

$$
P_{\theta}(A)=0 \text { for all } \theta \in \theta_{1} \text {. }
$$

That nonexistence of a splitting set is weaker than the connectedness property is seen from the following example.

EXAMPLE 4. Let $P$ be a family consisting of all two-point distributions and the standard normal distribution. Then $P$ is not connected and this does not possess a splitting set.

ACKNOWLEDGMENTS. Work of the first author is partially support by NSF Grant No. 79-04693.

\section{REFERENCES}

1. Basu, D. (1955). On statistics independent of a complete sufficient statistic. Sankhya A, 15, 337-380.

2. Basu, D. (1958). On statistics independent of sufficient statistic. Sankhya A, 20, 223-226.

3. Basu, D. and Ghosh, J.K. (1967). Sufficient statistics in sampling from a finite universe. Proc. 36th Session Internat. Statist. (In ISI Bulletin), 850-859.

4. Cheng, S.C. (1978). A Mathematical Study of Sufficiency and Adequacy in Statistical Theory. Ph.D. Thesis, submitted to the Florida State University.

5. Halmos, P. R. and Savage, L. J. (1949). Applications of the Radon-Nikodym theorem to the theory of sufficient statistics. Ann. Math. Statist. 20, 225-241.

6. Hasegawa, M. and Perlman, M. D. (1974). On the existence of a minimal sufficient subfield. Ann. Statist. 2, 1049-1055.

7. Koehn, V. and Thomas, L. D. (1975). On statistics independent of a sufficient statistic: Basu's lemma. American Statistician, 29, 40-43.

8. Pathak, P. K. (1975). Note on Basu's lemma. Technical Report No. 308. The University of New Mexico.

9. Pitcher, T. S. (1957). Sets of measures not admitting necessary and sufficient statistics or subfield. Ann. Math. Statist. 28, 267-268.

10. Pitcher, T. S. (1965). A more general property than domination for sets of probability measures. Pacific J. Math. 15, 597-611. 


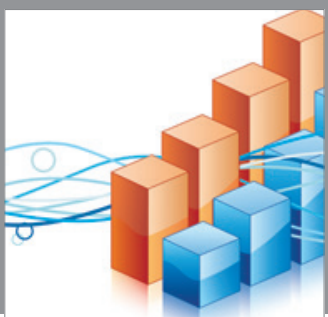

Advances in

Operations Research

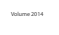

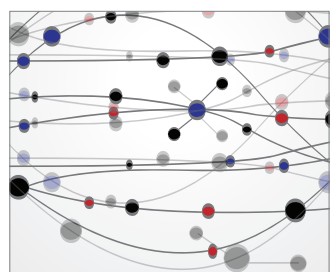

\section{The Scientific} World Journal
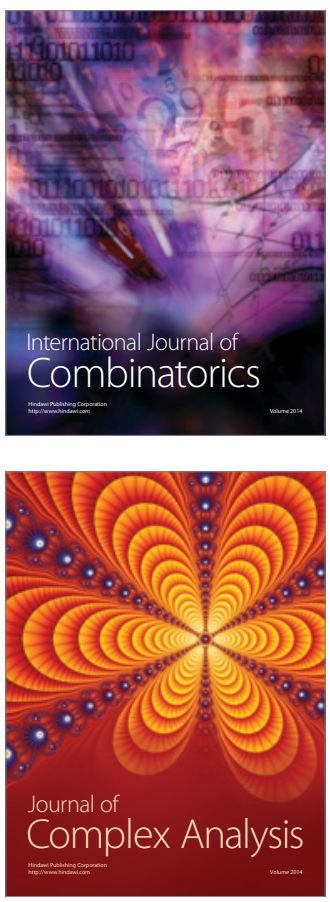

International Journal of

Mathematics and

Mathematical

Sciences
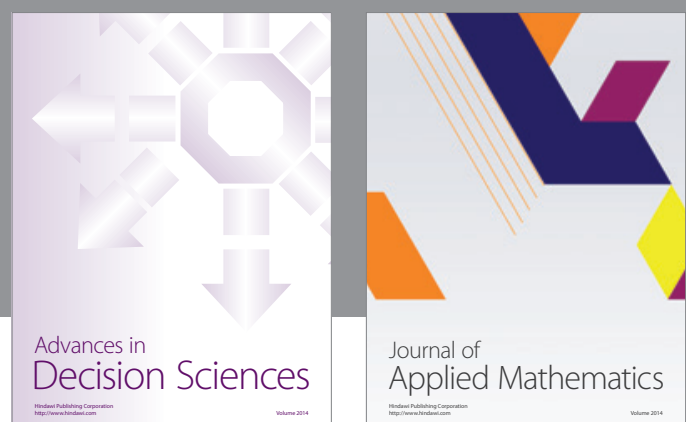

Journal of

Applied Mathematics
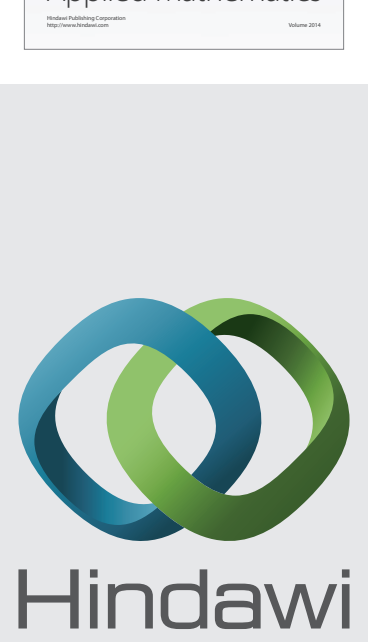

Submit your manuscripts at http://www.hindawi.com
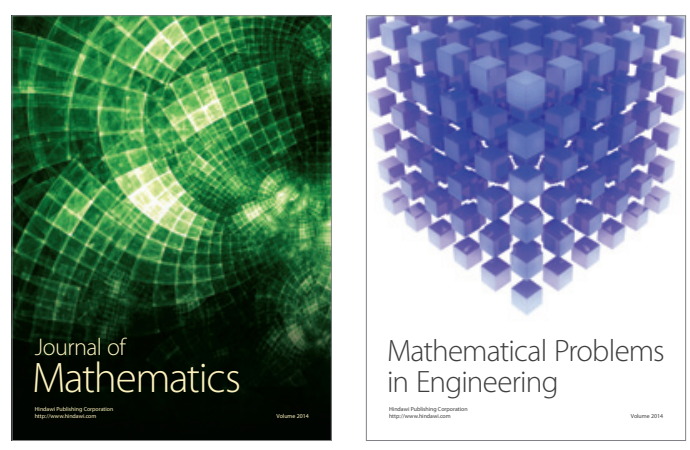

Mathematical Problems in Engineering
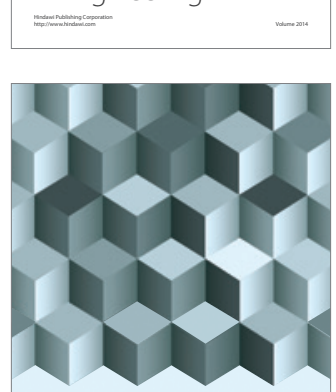

Journal of

Function Spaces
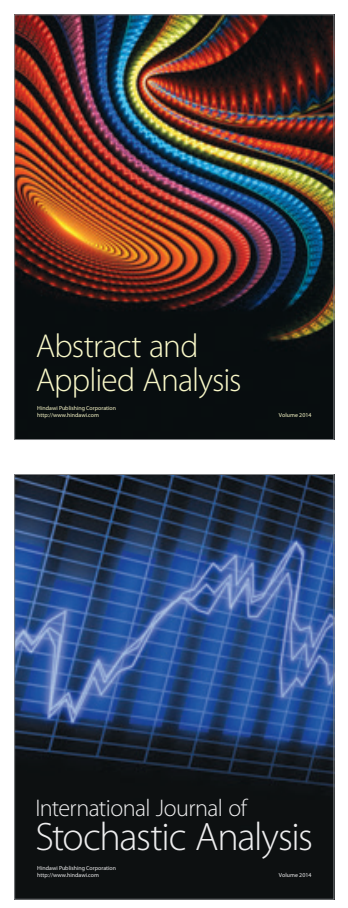

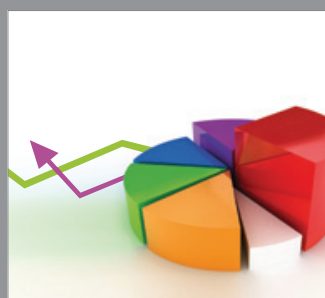

ournal of

Probability and Statistics

Promensencen
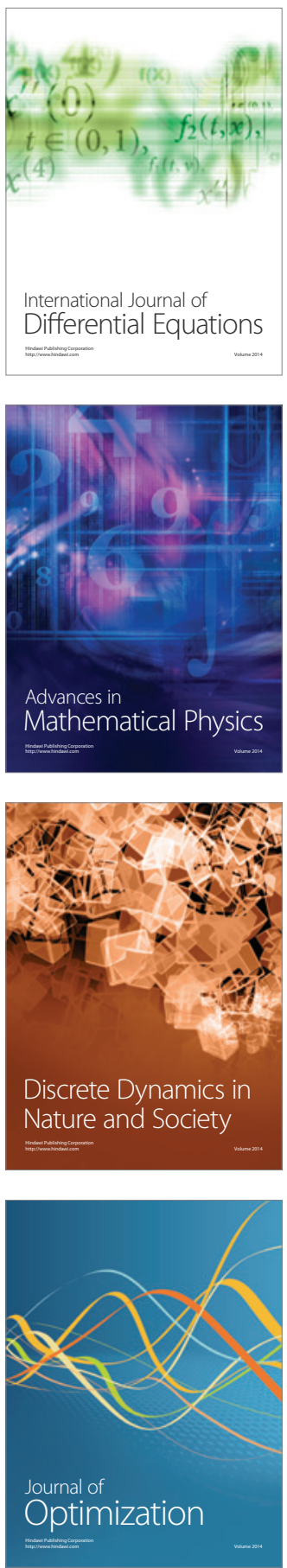